\title{
Actualización sobre endocarditis infecciosa en válvula protésica.
}

\author{
Nathalia Badilla Aa, Ricardo Zalaquett S. \\ Departamento de Enfermedades Cardiovasculares, Facultad de Medicina. \\ Pontificia Universidad Católica de Chile. \\ alnterna de $7^{\circ}$ año de Medicina \\ Recibido el 21 de septiembre de 2009, aceptado el 30 de octubre de 2009
}

\section{Resumen}

Rev Chil Cardiol 2009; 28: 414-420

La endocarditis en válvulas protésicas es aún tema de controversia, en especial en referencia al tipo de tratamiento que se debe elegir. Debido a que los conocimientos actuales sobre esta patología están basados principalmente en la literatura y, por ende, en sus variados exponentes (experiencias clínicas, revisiones sistemáticas, guía clínicas de diferentes sociedades de cardiología, estudios observacionales, entre otros), es necesario hacer una actualización continua de la información. Esta revisión bibliográfica se centra en la incidencia, etiología, diagnóstico y tratamiento de la endocarditis protésica. En ella se llega la conclusión de que los principales microorganismos productores de esta patología son el Estafilococo aureus y el Estafilococo coagulasa-negativo. También se reconocen aquellos factores de riesgo para los cuales la cirugía es mandatoria, entre los que destacan las infecciones por S. aureus y la endocarditis protésica complicada, ya sea por insuficiencia cardíaca, alteración de la conducción, formación de abscesos y/o embolías recurrentes. De esta manera, con la información recopilada, se identificaron aquellos pacientes con opción a tratamiento médico, y aquellos con indicación de manejo quirúrgico. Para presentar mejor nuestras conclusiones, se construyeron esquemas de fácil consulta y lectura, para apoyar la decisión del tipo de manejo frente a una endocarditis en válvulas protésicas.

Palabras claves: Endocarditis, Válvulas protésicas.

\section{UP-Date in prosthetic valve endocarditis}

Prosthetic valve endocarditis is still a matter of controversy, especially the type of treatment that should be chosen for an individual patient. Since the current knowledge of this patology is based mainly on the literature and in its many and varied exponents (clinical experience, sistematic review, practice guideslines of cardiology associations, observational studies, etc), it is necessary to update the information continuously. This review is focused mainly in the incidence, etiology, diagnostics and treatment of prosthetic endocarditis. The principal microroganisms related to this patology are Staphylococcus aureus and coagulase-negative Staphyloccoco. Risk factors were identified for witch surgery is mandatory, such us Staphylococcus aureus infection and a complicated prosthetic valve endocarditis (cardiac failure, conduction abnormalities, abscess formation and recurrent embolism). With this information, patients that could have a medical treatment option can be recognized, as well as those who will require surgery. In order to present in a better way our conclusions, easily reading diagrams were included to facilitate the decision process when facing up a prosthetic valve endocarditis.

Key words: Endocarditis, Prosthetic valve

Correspondencia: Dr. Ricardo Zalaquett S.

Departamento de Enfermedades Cardiovasculares. Pontificia

Universidad Católica de Chile. Marcoleta367, $8^{\circ}$ piso.

Santiago de Chile- Cirugía Cardíaca

Teléfono: 02-3543231 - Fax: 02-6390108

Correo Electrónico: rzalaque@med.puc.cl 


\section{Introducción}

Gracias al avance de la medicina actual se ha logrado cambiar el curso de enfermedades que comprometían en forma significativa la calidad de vida de los pacientes. Una de ellas son las patologías cardiovalvulares, y, en particular, aquellas que requieren recambio o reparación valvular. De hecho, se estima que en Estados Unidos y Europa más de 150.000 intervenciones de este tipo son realizadas al año. Sin embargo, así como surgen nuevas técnicas y procedimientos, aparecen las complicaciones asociadas. Es así entonces, como la endocarditis infecciosa en válvulas protésicas (EVP) ha tomado cada vez más relevancia, representando un desafío tanto para médicos como para cirujanos. Este incremento está asociado, además, al creciente número de intervenciones intravasculares (marcapasos, desfibriladores, resincronizadores) y al uso de hemodiálisis, representando focos probables de infección1.

Esta infección es poco frecuente pero grave, lo que acentuado por su difícil diagnóstico, pronóstico ominoso y complicadas estrategias terapéuticas, conlleva un alto riesgo de morbi-mortalidad (20 a $80 \%)^{2}$.

La siguiente revisión aborda y analiza las últimas publicaciones en cuanto a su incidencia, etiología y factores de riesgo, aproximación diagnóstica y opciones de tratamiento.

\section{Incidencia}

En forma global la endocarditis infecciosa (EI) tiene una incidencia aproximada de 1,7 a 6,2 casos en 100.000 personas, correspondiendo el $15 \%$ a $20 \%$ a infecciones en válvulas protésicas 1,2,3,4. Afecta en igual proporción a ambos tipos de prótesis, mecánica y biológica 5,6 , cada una con un riesgo de $5,7 \%$ a 5 años (variando el rango entre $1 \%$ a $6 \%)^{2}$, aunque algunos estudios han registrado una mayor frecuencia de EVP tardía en válvulas biológicas 3,4 .

En este mismo contexto, el riesgo personal de los pacientes con recambio valvular de presentar una El es de $0,3-1,2 \%$ por año2,6.

Dentro de los factores de riesgo para adquirir esta enfermedad se encontró que las infecciones intrahospitalarias y/o comorbilidades que requerían manejo invasivo o de enfermería (por ejemplo hemodiálisis o quimioterapia) tenían una asociación de hasta $36 \%$ del total de los pacientes con endocarditis protésica, presentándose la mayoría $(70 \%)$ durante el primer año post-cirugía1.

\section{Etiopatogenia}

La endocarditis bacteriana tiende a ser diferenciada entre temprana o tardía, según el tiempo transcurrido desde la cirugía. Esto nos sitúa en distintos panoramas en cuanto a la etiología y por tanto a la agresividad del tratamiento.

El punto de corte actualmente usado entre una EVP precoz y tardía es de 60 días, desde la implantación de la prótesis. Sin embargo, en este mismo contexto, estudios basados en los microorganismos encontrados en hemocultivos, han estimado conveniente hacer esta división al año de la cirugía, ya que sólo transcurrido ese período de tiempo se hallaba clara diferencia entre los agentes productores de El2,7,8,9 .

La cepa estafilocósica es la que produce con mayor frecuencia esta patología8. Un estudio multicéntrico publicado en JAMA demostró una prevalencia de esta cepa de hasta un $36 \%$, siendo el Estafilococo aureus el principal causante de la forma precoz (35,9\%), y el Estafilococo coagulasa negativo de EVP tardía (19,9\%). Cabe mencionar que en este mismo estudio, la diferencia esencial del S. aureus como causante de enfermedad temprana o tardía lo marcaba el hecho de presentar resistencia a Meticilina (en EVP precoz era de 18\%, y en tardía era de $3 \%) 1$. Este último hallazgo también se encontró en un estudio realizado en Bélgica, en donde el S. Aureus era causante de un $29 \%$ de las EVP y la diferencia, en cuanto a resistencia a Meticilina, en una EVP precoz y tardía era, de $24 \%$ y $2 \%$, respectivamente 10 .

Por otra parte, en estudios realizados tanto en Japón como España, se identificó como primera causa de EVP, tanto temprana como tardía, a Estafilococo epidermidis 11,12 . En relación a este mismo agente, un estudio prospectivo multicéntrico realizado en 28 países, concluyó que a pesar que el Estafilococo coagulasa-negativo era la segunda causa de 
infecciones protésicas, estaba asociado a mayor riego de complicaciones valvulares, con mayor probabilidad de presentar un absceso intracardíaco, incluso que el $\mathrm{S}$. Aureus y el S. Viridans, representado por un $38 \%$, $23 \%$ y $20 \%$ respectivamente. Esto último, explicado por la demora del diagnóstico, ya que 1 de cada 5 pacientes con este microorganismo había cursado con un cuadro infeccioso por un mes antes del diagnóstico de EVP9.

Finalmente, el agente Estreptocócico adquiere importancia en una EVP tardía, teniendo un segundo lugar de causalidad, con un $25 \%$ de incidencia1,10. A parte de estos 2 grandes grupos bacterianos y sus diferentes especies, se han hallado otros agentes causantes de enfermedad que varían entre un estudio y otro. En este mismo estudio multicéntrico de JAMA, se encontró hasta una $24 \%$ de prevalencia de Enterococo en EVP precoz y $17 \%$ en tardía 1 .

\section{Diagnóstico}

Para diagnosticar una Endocarditis Bacteriana en válvulas protésicas se continúa usando los criterios de Duke, postulados en 199413. Basado en este mismo concepto, en una serie retrospectiva realizada en España, se observó que al incluir dos nuevos criterios menores (insuficiencia cardíaca y trastornos de conducción, ambos de reciente comienzo) aumentaba la sensibilidad para el diagnóstico de una EVP tardía de $82 \%$ a $97 \%$, sin tener significancia estadística para el diagnóstico de EVP precoz 5,12 .

El diagnóstico se basa en el hallazgo de hemocultivos positivos asociado a alteraciones anatómicas valvulares. Es por esto último que es esencial, además, el uso de imágenes. Dentro de ellos, el más utilizado es el ecocardiograma. El modo transtorácico posee una sensibilidad de $70 \%{ }^{2}$. La incorporación del ecocardiograma transesofágico ha permitido aumentar la sensibilidad a un $84 \%$, en especial para lesiones, vegetaciones y abscesos perivalvulares ${ }^{2}$. El principal limitante de estos avances tecnológicos es ser operador dependiente.

Como ayuda al diagnóstico a través de imágenes, existen casos reportados en la literatura donde se ha usado Tomografía Computarizada de Múltiples Cortes. Gracias a éste se objetivaron complicaciones en relación a la válvula protésica, confirmándose en algunos casos la sospecha de endocarditis. Estos estudios son, entonces, otro método no invasivo alternativos para la evaluación de una EVP y sus complicaciones asociadas 14,15 .

Sin embargo, aún teniendo criterios bien definidos para el diagnóstico, el escenario es complejo, debido a varios factores: Uno de ellos es la presentación atípica de este tipo de patología, especialmente en el post-operatorio inmediato, donde la mayoría de los pacientes presentan fiebre u oligosintomatología, sin necesariamente estar cursando una infección. Otro factor es el hecho de que los hemocultivos resultan negativos de un $20 \%$ a un $29 \%$ en EVP precoz y de $16 \%$ a un $45 \%$ en EVP tardía11,12 permaneciendo negativos hasta un $11 \%$ de los cultivos repetidos ante la sospecha clínica2 ${ }^{2}$.

También se ha encontrado en EVP menor presencia de soplos cardíacos en comparación con infecciones en válvulas nativas ${ }^{16}$.

Es en este escenario, entonces, donde adquiere vital importancia el grado de experiencia que posee el clínico a cargo. Por lo tanto, se debe poner siempre prioridad a la sintomatología, y, por tanto, la alta sospecha diagnóstica del médico tratante es mandatoria para repetir hemocultivos y ecocardiogramas cuantas veces se estime necesario.

\section{Pronóstico}

A pesar de ser una enfermedad poco frecuente, la endocarditis protésica tiene un riesgo importante de morbi-mortalidad. Dentro de las complicaciones probables se encuentra el daño valvular, la insuficiencia cardíaca secundaria, alteraciones de la conducción y embolias a distancia, siendo la muerte la más temida, con una incidencia que alcanza entre $20 \%$ y $30 \% 1,6$. Por ello es perentorio identificar grupos de alto riesgo, para los cuales el tratamiento debe ser precoz y agresivo. Diferentes estudios han coincidido en una serie de factores que aumentarían el riesgo de la mortalidad, y, por lo tanto, es en los pacientes que los presentan donde se debería tomar una decisión rápida de conducta que, por lo general, implica cirugía. Estos factores se detallan en la tabla 1. 
Tabla 1: Factores que han sido asociados con aumento de la mortalidad en endocarditis bateriana en válvulas protésicas 1,2,3,6,11,1213,16

1. Edad avanzada

2. Infección asociada con cuidados especiales realizadas por equipo de salud.

3. Comorbilidad grave

4. Procedimiento intravascular.

5. Endocarditis por Staphylococcus Aureus

6. Complicaciones secundarias a EVP:
a. Insuficiencia Cardíaca Congestiva
b. Accidente vascular cerebral
c. Absceso perivalvular
d. Bacteremia persistente.
e. Alteración de la conducción eléctrica.
7. Complicación cerebral.
8. Endocarditis en válvula protésica precoz
9. Mediastinitis
10. Shock séptico
11. Insuficiencia renal

En estos grupos de alto riesgo se vio que el poseer una infección por Estafilococo aureus y una EVP complicada, eran los factores de peor pronóstico, teniendo un riesgo de mortalidad muy alto, llegando incluso hasta OR: 13.7 y OR: 6.4 , respectivamente $1,3,6,10$. Un estudio reciente multicéntrico realizado con datos de 62 centros de salud en 28 países, demostró que aquellos pacientes requerientes de cuidados de enfermería y/o procedimientos invasivos (como hemodiálisis, quimioterapia intravenosa, cuidados de herida, entre otros) tenían una asociación con el desarrollo de EVP. Este tipo de pacientes representó hasta un $36.5 \%$ del total de endocarditis protésica, siendo de éstos un $69.5 \%$ causados por enfermedad nosocomial1 1 .

\section{Tratamiento}

El tratamiento de la endocarditis depende tanto de los factores de riesgo del paciente como de las características del cuadro que este cursando. Las 2 alternativas de tratamientos actuales son el tratamiento médico y el quirúrgico, pero aun se mantiene la gran disyuntiva sobre cuando elegir a uno $u$ otro. El manejo médico consiste en la utilización de antibióticos y monitorización en una unidad de cuidados especiales, por lo menos las primeras semanas, para pesquisar complicaciones en forma precoz. El manejo quirúrgico implica un nuevo recambio valvular para controlar la infección, eliminando tejido necrótico e infectado, y en la corrección de las disfunciones valvulares. La incidencia actual de cirugía para tratar una endocarditis protésica es de un $0,5 \%$ al año $(0,3 \%-0,8 \%)^{17}$.

Estudios actuales, que comparan tratamientos y resultados de sobrevida, han logrado diferenciar un subgrupo de pacientes con claro y mandatorio requerimiento de cirugía. Entre estos, un estudio multicéntrico de 104 casos, concluyó que no había diferencia estadísticamente significativa en la sobrevida de paciente estables tratados en forma médica 0 quirúrgica ( $17 \%$ y $25 \%$, respectivamente), pero la mortalidad sí se veía reducida por un abordaje quirúrgico en pacientes con factores de riesgo, sobresaliendo la EVP precoz y la causada por S. Aureus ${ }^{3}$. Otro estudio hecho en Bélgica, realizó una división parecida, separando en 3 grupos a sus pacientes: el primero fue de absoluto manejo médico, representado por pacientes estables y sin descompensación. Se obtuvo una mortalidad a 6 meses de $4 \%$. El siguiente grupo fue de manejo médico-quirúrgico, teniendo 
pacientes con comorbilidades descompensadas, y/o con una EVP complicada. Su mortalidad fue de $27 \%$, claramente superior al grupo anterior. Por último, el tercer grupo se manejó sólo con medicamentos y monitorización pues tenían contraindicación de tratamiento quirúrgico. Su mortalidad fue de un $75 \%$. En términos de comparación, este último grupo no es de utilidad pues la baja sobrevida era de esperarse, ya que eran pacientes muy descompensados y con comorbilidades, en que el manejo conservador era evidentemente insuficiente ${ }^{10}$.

En otro estudio prospectivo, realizado en Japón, se identificó que el principal riesgo de mortalidad asociada al tratamiento quirúrgico radicaba en 2 factores: si se trataba de una EVP precoz y/o si la cirugía era de emergencia. No se encontró relación con la edad, sexo, posición de la válvula o antecedentes de El previa11.
En relación a esto último, un estudio retrospectivo realizado en Inglaterra a más de 54.000 pacientes que requirieron cirugía debido a una endocarditis protésica, concluyó que la tasa de sobrevida a 30 días de la cirugía era de $80 \%$, con una disminución a un $67 \%$ al año, $55 \%$ a los 5 años y $38 \%$ a los 10 años. En este último estudio sí se encontró como factor determinante la edad del paciente en todos los rangos de tiempo. No había relación de causalidad con el sexo ni con el tipo de válvula protésica infectada ${ }^{17}$.

De estos trabajos se concluye que la decisión terapéutica debe ser individualizada, basándose en los factores de riesgo que presente cada paciente. En las Tablas 2 y 3 se agrupan estas características de manera de esquematizar los tratamientos sugeridos por distintas guías internacionales, según las características de los pacientes.

Tabla 2: Guías para decisión de tratamiento médico $1,2,3,6,7,10$,

\section{Tratamiento médico:}

1. Endocarditis protésica sin complicaciones

2. Endocarditis con microorganismos diferente a Staphylococcus aureus

3. En aquellos pacientes con posibilidad de un seguimiento continuo, reevaluación con hemocultivos y ecocardiograma transesofágico.

Tabla 3: Guías para decisión de tratamiento quirúrgico $1,2,3,6,7,10,18$

\section{Tratamiento quirúrgico}

1. Endocarditis protésica precoz (menos de 12 meses posterior a recambio valvular)

2. Endocarditis protésica complicada:
a. Insuficiencia Cardiaca
b. Dehiscencia evidenciada por imagenología
c. Formación de absceso
d. Aumento de la obstrucción producido por una vegetación o regurgitación
e. Anormalidades en la conducción eléctrica cardiaca

3. Evidencia de bacteremia persistente o émbolos recurrentes a pesar de un adecuado tratamiento antibiótico (durante 7-10 días)

4. Microorganismos aislado: Staphylococcus aureus

5. Se podría considerar en aquellos con infección recurrente.

6. Necesidad de recambio según apreciación de cirujano cardiovascular con experiencia en el tema. 


\section{Conclusión}

La endocarditis en válvulas protésicas es una complicación grave del recambio valvular. El Estafilococo aureus continúa siendo el principal causante de la endocarditis protésica.

Debido a lo atípico del cuadro de presentación, todo clínico que esté en contacto con este tipo de pacientes debe sospecharla, buscarla dirigidamente y sobre todo identificar aquellos casos donde se cumplen los criterios de gravedad. Una endocarditis que se complicó, una EVP en pacientes con manejo invasivo y/o de enfermería frecuente y enfermos en los que se aisló como agente causal un S. aureus, representan la población con más alto riesgo de morbi-mortalidad, para los cuales el recambio valvular es indiscutible.

El tratamiento de la endocarditis en válvulas protésicas sigue siendo un tema de discusión. Mundialmente es aceptado tanto el manejo médico como quirúrgico, pero la elección entre ambos debe ser determinada en forma particular para cada paciente.

Actualmente existe evidencia a favor del tratamiento médico en primera instancia, pero sigue siendo recomendada la cirugía precoz en pacientes de alto riesgo.

\section{Referencias}

1. WANG A, ATHAN E, PAPPAS PA, FOWLER VG JR, OLAISON L, PARE C, et al. Contemporary clinical profile and outcome of prosthetic valve endocarditis. International Collaboration on Endocarditis-Prospective Cohort Study Investigators. JAMA 2007; 297:1354-61

2. HABIB G, THUNY F, AVIERINOS JF. Prosthetic valve endocarditis: current approach and therapeutic options. Prog Cardiovasc Disc 2008; 50: 274-81.

3. HABIB G, TRIBOUILLOY C, THUNY F, GIORGI R, BRAHIM $A, A M A Z O U Z M$, et al. Prosthetic valve endocarditis: who needs surgery? A multicenter study of 104 cases. Heart 2005; 91:9 54-9.

4. FEDORUK LM, JAMIESON WR, LING H, MACNAB JS, GERMANN E, KARIM SS, et al. Predictors of recurrence and reoperation for prosthetic valve endocarditis after valve replacement surgery for native valve endocarditis. J Thorac Cardiovasc Surg 2009; 137: 326-33

5. ZALAQUETT R, ÁLVAREZ H. Endocarditis infecciosa de válvula protésica Rev. Chilena de Cirugía 2003; 55: 548-553.

6. AKOWUAH EF, DAVIES W, OLIVER S, STEPHENS J, RIAZ I, ZADIK P, et al. Phosthetic valve endocarditis: early and late outcome following medical or surgical treatment. Heart 2003; 8: 269-272.

7. HORSTKOTTE D, FOLLATH F, GUTSCHIK E, LENGYEL M., OTO A., PAVIE A, et al. Guidelines on prevention, diagnosis and treatment in infective endocarditis executive summary; the task force on infective endocarditis of the European Society of Cardiology. Eur Heart J 2004; 25: 267-76

8. LOPEZ J, REVILLA A, VILLACOSTA I, VILLACORTA E, GONZÁLEZ-JUANATEY C, GÓMEZ I, et al Definition, clinical profile, microbiological spectrum and prognostis factors of $\mathrm{e}$ arly-onset prosthetic valve endocarditis. Eur Heart J 2007; 28: 760-765

9. CHU VH, MIRO JM, HOEN B, CABELLL CH, PAPPAS PA,
JOMES $\mathrm{P}$, et al. Coagulasa -negative staphylococcl prosthetic valve endocarditis a contemporary update based on the International Collaboration on Endocarditis: prospective cohort study. Heart 2009; 95: 570-576.

10. HILL EE, HERREGODS MC, VANDERSCHUEREN S, CLAUS $P$, PEETERMANS WE, HERIJGERS $P$. Management of prosthetic valve infective endocarditis. Am J Cardiol. 2008; 101: 1174-8.

11. SASAKI Y, ISOBE F, KINUGASA S, IWATA K, NAGAMACHI $\mathrm{K}, \mathrm{KATO} Y$, et al. Early and late outcomes after reoperation for prosthetic valve endocarditis. Jpn J Thorac Cardiovasc Surg 2001; 49: 224-9

12. PÉREZ-VÁSQUEZ A, FARIÑAS MC, GARCÍA-PALOMO JD, BERNAL JM, REVUELTA JM, GONZÁLEZ-MANCIAS J. Evaluation of the Duke criteria in 93 episodes of prosthesic valve endocarditis. Arch Intern Med 2000 24; 160: 1185-91.

13. DURACK DT, LUKES AS, BRIGHT DK. New criteria for diagnosis of infective endocarditis: utilization of specific echocardiographic findings. Duke Endocarditis Service. Am J Med 1994; 96: 200-9

14. KIM RJ, WEINSAFT JW, CALLISTER TQ, MIN JK. Evaluation of prosthetic valve endocarditis by 64-row multidetector computed tomography.Int J Cardiol. 2007; 120: 27-9.

15. TSAI IC, LIN YK, CHANG Y, FU YC, WANG CC, HSIEH SR, et al. Correctness of multi-detector-row computed tomography for diagnosing mechanical prosthetic heart valve disorders using operative findings as a gold standard. Eur Radiol 2009; 19: 857-67

16. GUERRERO F, LOPEZ J, GOYONECHEA A, FRAILE J, GÓRGOLAS M. Endocarditis caused by Staphylococcus aureus: A reappraisal epidemiologic, clinical, and Pathologic manifestations with analysis of factors determining outcome. Medicine 2009; 88: 1-22

17. EDWARD MB, RATNATUNGA CHP, DORE CJ. Thirty-day 
Actualización sobre endocarditis infecciosa en válvula protésica.

mortality and long-term survival following surgery for prosthetic endocarditis: a study from the UK heart valve registry. Eur J Cardiothorac Surg 1998; 14: 156-164.

18. BONOW R, CARABELLO B, CHATTERJEE M, DE LEON A., FAXON D., FREED M., et al. ACC/AHA 2006 Guidelines for the Management of Patients With Valvular Heart Disease: A Report of the American College of Cardiology/American
Heart Association Task Force on Practice Guidelines (Writing Committee to Revise the 1998 Guidelines for the Management of Patients With Valvular Heart Disease) Developed in Collaboration With the Society of Cardiovascular Anesthesiologists Endorsed by the Society for Cardiovascular Angiography and Interventions and the Society of Thoracic Surgeons. J Am Coll Cardiol 2006; 48: 1-148. 